\title{
Cognitive, behavior and academic functioning in adolescent and young adult survivors of childhood acute lymphoblastic leukemia: A report from the Childhood Cancer Survivor Study
}

\author{
Lisa M. Jacola, PhD ${ }^{1}$, Kim Edelstein, PhD $^{5}$, Wei Liu, PhD $^{2}$, Ching-Hon Pui, MD ${ }^{3}$, Robert \\ Hayashi, MD ${ }^{6}$, Nina S. Kadan-Lottick, MD7, Deokumar Srivastava, PhD², Tara Henderson, \\ $\mathrm{MD}^{8}$, Wendy Leisenring, $\mathrm{ScD}^{9}$, Leslie L. Robison, $\mathrm{PhD}^{4}$, Gregory T. Armstrong, $\mathbf{M D}^{4}$, and \\ Kevin R. Krull, $\mathrm{PhD}^{1,4}$ \\ ${ }^{1}$ Department of Psychology, St. Jude Children's Research Hospital, Memphis TN 38105-3678 \\ ${ }^{2}$ Department of Biostatistics, St. Jude Children's Research Hospital, Memphis TN 38105-3678 \\ ${ }^{3}$ Department of Oncology, St. Jude Children's Research Hospital, Memphis TN 38105-3678 \\ ${ }^{4}$ Department of Epidemiology and Cancer Control, St. Jude Children's Research Hospital, \\ Memphis TN 38105-3678 \\ ${ }^{5}$ Pencer Brain Tumor Centre, Princess Margaret Cancer Center, Toronto ON M5G 2M9, Canada \\ ${ }^{6}$ Department of Oncology, Washington University at St. Louis, St. Louis MO 63130 \\ ${ }^{7}$ Yale Section of Pediatric Hematology-Oncology and Yale Cancer Center, New Haven CT 06510 \\ ${ }^{8}$ Department of Oncology, University of Chicago, Chicago IL 60637 \\ ${ }^{9}$ Clinical Research and Public Health Sciences Division, Fred Hutchinson Cancer Research \\ Center, Seattle WA 98109
}

\section{Abstract}

Background-Survivors of childhood acute lymphoblastic leukemia (ALL) are at risk for neurocognitive deficits that impact adolescence and young adulthood development, and influence educational attainment and future independence.

\begin{abstract}
Methods-Cognitive and behavior symptoms were assessed via a standardized parent questionnaire for 1560 adolescent survivors of ALL diagnosed between 1970 and 1999. Significant symptoms ( $\geq 90^{\text {th }}$ percentile) and learning problems were compared between survivors and a sibling cohort. Multivariable regression models were used to examine associations with demographic/treatment characteristics. In a subset of survivors with longitudinal data ( $n=925)$, associations between adolescent symptoms/problems and adult educational attainment were examined.
\end{abstract}

\footnotetext{
Declaration of interests: no conflicts exist

Author Contributions

Study design, manuscript writing, and final approval of the manuscript: All authors

Data assembly and collection: WL, LLR, GTA

Data analysis and interpretation: LMJ, WL, DS, WL, LLR, GTA, KRK
} 
Findings-Compared to siblings, survivors treated with chemotherapy only were more likely to demonstrate headstrong behavior ( $19 \%$ vs. $14 \%, \mathrm{P}=0.010)$, inattention-hyperactivity ( $19 \%$ vs. $14 \%, \mathrm{P}<0.0001)$, social withdrawal (18\% vs. $12 \%, \mathrm{P}=0.002)$, and had higher rates of learning problems ( $28 \%$ vs. $14 \%, \mathrm{P}<0.0001)$. In multivariable models among survivors, increased cumulative dose of intravenous methotrexate (i.e., $>4.3 \mathrm{~g} / \mathrm{m}^{2}$ ) conferred higher risk for inattentionhyperactivity (Relative Risk [RR] 1.53, 95\% Confidence Interval [CI] 1.13-2.08) and special education (RR 2.04, 95\% CI 1.41-2.93). Adolescent survivors with cognitive or behavior problems and those with learning problems were less likely to graduate from college as young adults.

Interpretation-Though modern therapy for childhood ALL has eliminated the use of CRT, adolescent survivors treated with only chemotherapy remain at increased risk for cognitive, behavior, and academic problems that adversely impact adult education outcomes. Interventions to improve neurobehavioral functioning should be prioritized, particularly those that target inattention-hyperactivity and learning problems.

Acute lymphoblastic leukemia (ALL) is the most common childhood malignancy, accounting for $26 \%$ of all new childhood cancer diagnoses. (1) With current 5-year survival rates above $90 \%$ (2), a primary objective of contemporary therapy includes reducing treatment late effects while maintaining high cure rates. Historically, treatment modifications have included replacing cranial radiation therapy (CRT) with intensified systemic and intrathecal (IT) chemotherapy for CNS prophylaxis. (3) The majority of studies showed that replacement of CRT with chemotherapy improved global cognitive outcomes, and resulted in less acute and long-term neurotoxicity in survivors of childhood ALL. $(4,5)$ Still, studies in survivors treated without CRT have suggested increased risk for deficits in attention, processing efficiency, and executive functions $(4,5)$ that are associated with reduced quality of life. (6) Currently, risk factors for neurocognitive difficulties include greater intensity of CNS-directed chemotherapy, $(7,8)$ younger age at diagnosis, (9-11) and female gender. (11, 12) However, existing studies are limited by small sample sizes, treatment era effects, single site data collection, and cross-sectional study design. Multi-site studies with large cohorts treated without CRT and followed longitudinally are needed to quantify the impact of contemporary therapy on neurocognitive outcomes.

Adolescence is a critical developmental period that involves substantial biological, psychological, and social changes. $(13,14)$ The impact of neurocognitive deficits on daily function may increase during adolescence, given higher expectations for planning, organization, and self-direction (i.e. executive functions). Adolescent survivors with neurocognitive deficits frequently engage in poor health behaviors (e.g., smoking) that are likely to continue into adulthood. It is reasonable to hypothesize that cognitive and behavior problems present in adolescents may predict poor adult outcomes.

The current study used data from the Childhood Cancer Survivor Study (CCSS) to identify the prevalence and predictors of parent-reported cognitive, behavior, and learning problems in adolescent survivors of ALL. Studies of psychological functioning in siblings of childhood cancer survivors suggest that, compared to the general population, these children are at increased risk for acute and long-term psychological difficulties. (15) As such, we compared the prevalence of problems in survivors to a sibling comparison group of similar 
age. We hypothesized that (1) difficulties would be more prevalent in adolescent survivors versus siblings of similar age and (2) cognitive, behavior, and learning problems in survivors would be positively associated with younger age at diagnosis, female gender, and greater treatment intensity. Our second objective was to examine the association between problems in adolescent survivors and adult education attainment.

\section{Methods}

The CCSS is a multi-institutional, retrospective cohort study designed to evaluate long-term impact of childhood cancer and treatment on health and functional outcomes. $(16,17)$ To be eligible for inclusion in the study, survivors must have been treated for childhood cancer at 1 of 31 institutions and survived for at least 5 years after diagnosis. Participants completed a 24-page baseline questionnaire that included sociodemographic information, medical history, and functional limitations. Baseline data was collected in 1992 for survivors diagnosed between 1970 and 1986 and in 2008 for survivors diagnosed between 1987 and 1999. Baseline data was collected between 1996 and 2005 from comparison siblings of survivors diagnosed with cancer between 1970 and 1986. Late effects were prospectively assessed through follow-up surveys completed between 2004 or 2007 for the survivors diagnosed between 1987 and 1999. The current analysis includes all survivors of childhood ALL and siblings whose parents completed the baseline survey when the survivor or sibling was 12-17 years of age. A secondary analysis of longitudinal outcomes was limited to survivors diagnosed between 1970 and 1986 who completed subsequent follow-up surveys (Figure 1). The human subjects committee at each collaborating institution approved the study protocol and all study procedures. Survivors or their proxy (for participants < age 18) gave consent for participation and release of medical records. Data from the following surveys was used in this study: Original Cohort: Baseline $<18$, Baseline Sibling $<18$, Follow-Up 2003, Follow-Up 2007; Expansion Cohort: Baseline < 18 - expanded. All survey materials are available at http://www.stjude.org/CCSS.

\section{Outcome Variables}

Parent ratings of cognitive and behavior problems were collected with the Behavior Problem Index (BPI), a standardized questionnaire that has been normed on a large nationally representative sample. (18) Factor analyses in the normative sample resulted in five domains: Antisocial, Anxiety-Depression, Headstrong (e.g., "Argues too much"), InattentionHyperactivity (e.g., "Difficulty concentrating"), and Social Withdrawal. For the current study, impairment was defined as having a domain score $\geq 1.3$ standard deviations above $\left(90^{\text {th }}\right.$ percentile) the mean score for the sibling comparison group. Special education services beginning after diagnosis (yes/no) was used as an index of learning problems in school. Selfreported adult education attainment was utilized for participants who completed longitudinal follow-up surveys at $\geq 25$ years of age and was categorized into: less than high school (one to twelve years), completed high school/obtained general education development (GED) certificate, training after high school or some college, and at least college graduate. 


\section{Independent variables}

Treatment exposures were abstracted from medical records. Occurrence of epilepsy and/or stroke with onset after cancer diagnosis was also evaluated, as these may impact adolescent and adult outcomes.

\section{Statistical Analysis}

Demographic and treatment variables were characterized using descriptive statistics. All analyses accounted for varying sampling probabilities using inverse probability of sampling as analytic weights. Frequency and summary statistics were compared between survivors treated with and without CRT and between survivors completing follow-up and those lost to follow-up by Chi-Square test or two-sample t-test. The frequency of impairment for each BPI domain and the rate of special education were compared (Chi-Square test) between all survivors and siblings, survivors treated without CRT and siblings, and survivors treated with versus without CRT. Univariate Poisson regression was used to estimate the relative risk for impairment on each BPI domain and for special education in the overall group of survivors compared to siblings. Reported results are not adjusted for intra-family correlation, as only $11 \%$ of the survivors had a sibling included in the analysis, and sensitivity analyses indicated no difference in results with adjustment versus without. Within the overall survivor cohort, separate multivariable Poisson regression models were used to estimate the relative risk (RR) and corresponding 95\% confidence intervals (CI) of impairment on each of the BPI domains and RR for special education, accounting for the following covariates of interest: age at diagnosis, sex, CRT (None, <20 Gy, $\geq 20$ Gy), IV methotrexate (MTX; None, $<4 \cdot 3 \mathrm{~g} / \mathrm{m}^{2}, \geq 4 \cdot 3 \mathrm{~g} / \mathrm{m}^{2}$ ), IT-MTX ( $<230 \mathrm{mg}, \geq 230 \mathrm{mg}$ ), and neurologic events. Cutoffs for treatment variables were chosen based on a median split, and are consistent with doses that separate low from standard/high risk arms on more contemporary chemotherapy-only protocols.'(19) To remove the confounding effect from CRT, additional multivariable models were conducted in the cohort of survivors treated without CRT. Univariate Poisson regression was used to examine the associations between impairment in each BPI domain and special education separately for the cohort of survivors treated with or without CRT. Analyses examining adult outcomes were restricted to survivors and siblings with available follow-up data and who were $\geq 25$ years old, in order to allow enough time for participants to graduate from college. Univariate Poisson regression models were used to examine the association between adolescent problems and adult educational attainment, dichotomized as "college graduation" and "no college graduation". All analyses were conducted in SAS 9.3 (SAS Institute, Cary, NC).

Data were not available to allow for direct comparison between the sibling cohort and normative data for the BPI factors. In order to compare siblings with normative data and to establish the representativeness of our sibling comparison group, we constructed two composite factors using a previously established approach: combining the AnxietyDepression and Social Withdrawal into an Internalizing Composite and the Antisocial, Headstrong, and Inattention-Hyperactivity into an Externalizing Composite. (18) There was no significant difference between the proportion of siblings with Externalizing problems (9.9\%) compared to the normative data $(10 \%, \mathrm{P}=0.90)$; however, a significantly greater proportion of siblings were rated as having Internalizing problems $(16 \cdot 8 \%, \mathrm{P}<0 \cdot 0001)$. 


\section{Role of the Funding Source}

The sponsors of the study had no role in study design, data collection, analysis, and interpretation, or writing of the report. The corresponding author (LMJ) had full access to study data and had final responsibility for the decision to submit for publication.

\section{Results}

Of the 6161 survivors of ALL in the CCSS, 1560 were adolescents at the time of the baseline survey; data were available for 1453 (93\%; Figure 1). The sibling comparison group consisted of 611 siblings (mean age $=15 \cdot 4$ ). Survivors were, on average, 3.7 years old at diagnosis and 15.3 years old at baseline survey (Table 1). The majority of survivors were White and males. Fifty-four percent of survivors were treated without CRT. There were no differences between survivors treated with or without CRT with regard to gender, race, ethnicity, age at diagnosis, time since treatment, and age at baseline survey. Survivors treated without CRT were more likely to receive higher cumulative doses of IT-MTX and IV-MTX. Survivors treated with CRT were more likely to receive treatment including anthracycline, alkylating agents, or dexamethasone and were more likely to report experiencing seizures or strokes following diagnosis.

We examined the prevalence and predictors of parent-reported problems in the adolescent survivors. Table 2 depicts the frequency of cognitive, behavior, and academic problems in survivors and siblings. The questionnaire from which information regarding education placement was derived asks parents to indicate the reason for placement in a special education program. Parents are given the following options, which are not mutually exclusive: missed school, low scores on tests, problems learning or concentrating, emotional or behavioral problems. Difficulties with learning/concentration and low test scores were the most common reasons endorsed (Supplementary Table 1). Compared to siblings, the overall survivor group had a significantly higher frequency of parent-rated problems in AnxietyDepression, Headstrong, Inattention-Hyperactivity, and Social Withdrawal. Among those survivors treated without CRT, frequencies of parent reported problems were significantly elevated across the following domains when compared with siblings: Headstrong, Inattention-Hyperactivity, and Social Withdrawal. Compared to those treated without CRT, survivors treated with CRT had significantly higher frequency of problems in AnxietyDepression, Inattention-Hyperactivity, and Social Withdrawal. Regardless of treatment, survivors were more likely to be placed in special education than siblings. At Baseline, the parents of survivors report on household income and insurance status, two frequently used indices of socioeconomic status. Compared to siblings, a significantly greater frequency of survivors were from a household with a reported income of less than $\$ 40,000$ (30.98 vs. $40.20 \%, \mathrm{P}=0.0001)$. There was no significant difference in the distribution of insurance status between survivors and siblings. The inclusion of household income and insurance status did not change the significant differences between survivors and siblings (Table 3).

In multivariable models, treatment with CRT $<20 \mathrm{~Gy}$ as compared to no CRT was associated with increased risk for problems in Anxiety-Depression, Inattention-Hyperactivity, Social Withdrawal, and increased risk for special education (Table 3). Compared to no CRT, exposure to $\geq 20 \mathrm{~Gy}$ CRT also increased risk for problems with Inattention-Hyperactivity and 
Social Withdrawal, as well as for special education placement. Treatment with higher cumulative doses of IV-MTX, relative to no IV-MTX exposure, conferred increased risk for problems with Inattention-Hyperactivity. Survivors treated with higher doses of IT-MTX ( $\geq$ $230 \mathrm{mg}$ ) were more likely to be in special education compared to those treated with lower doses $(<230 \mathrm{mg})$. Finally, the onset of a neurologic condition following diagnosis increased risk for Anxiety-Depression, Inattention-Hyperactivity, Social Withdrawal, and special education placement. There was no significant association between treatment intensity (IVMTX) and BPI outcomes or special education in multivariable models restricted to survivors treated without CRT (Supplementary Table 2). In univariate analyses stratified by CRT exposure, impairment on each of the five BPI factors was significantly associated with increased risk for special education compared to survivors without impairment on the BPI (Supplementary Table 3). Notably, problems on Inattention-Hyperactivity conferred the greatest risk for special education in survivors treated with $(\mathrm{RR}=1.93,95 \% \mathrm{CI} 1.62-2.31)$ or without CRT (RR=3·54, 95\% CI 2.77-4.52).

Longitudinal follow-up data was available for 925 (79\%) of the 1170 eligible survivors (Figure 1) and these data were used to examine patterns and predictors of education outcomes. Demographic and clinical characteristics for survivors with completed follow-up and those lost to follow-up are detailed in Supplementary Table 4. Participants completing follow-up were more likely to be White $(86.1 \mathrm{vs.} 70.9 \%, \mathrm{P}<0.001)$ and to be treated with lower cumulative doses of IT-MTX ( 48.7 vs. $37 \cdot 8 \%, \mathrm{P}=0.008$ ). When compared to participants with completed follow-up, those participants lost to follow-up were significantly more likely to have had problems with Antisocial Behavior at baseline (23.8 vs. $11.7 \%$, $\mathrm{P}<0 \cdot 001)$.

Analyses investigating adult educational outcomes were restricted to the 691 survivors and 259 siblings who were $\geq 25$ years old at follow-up, in order to allow enough time to lapse for survivors to feasibly attain the highest level of educational outcome (Supplementary Figure 1). We found significantly lower college graduate rates between survivors treated with CRT compared to siblings ( 43.0 vs. $65 \cdot 0, \mathrm{P}=<0.001)$ and between siblings and survivors treated without CRT (43.0 vs. $53 \cdot 0, \mathrm{P}=0.016)$ Univariate analyses showed that InattentionHyperactivity and special education placement during adolescence significantly increased risk of not graduating from college regardless of whether survivors received CRT (Figure 2).

\section{Discussion}

In this large study spanning three decades of diverse therapeutic protocols, adolescent survivors of ALL whose treatment did not include CRT demonstrate cognitive, behavior, and learning problems at a rate significantly lower than that observed in those treated with CRT, reflecting the relative preservation of neurobehavioral outcomes in survivors treated with contemporary therapy. Nonetheless, we found that a significant proportion of survivors treated without CRT still experience problems with headstrong behavior, inattentionhyperactivity, and social withdrawal. These problems were associated with increased risk for special education placement and predicted reduced adult educational attainment. Strengths of this study include the large size of the cohort, well-characterized treatment data, and the 
long duration of follow-up. Further, data on cognitive and behavior problems in adolescents were obtained via standardized ratings with documented reliability and validity.

Even with contemporary therapy, the prevalence of adverse outcomes in adolescent survivors of ALL remains substantial. Parent ratings document elevated levels of inattentionhyperactivity and headstrong behavior in 19\% of survivors treated without CRT, and one in four survivors treated without CRT was placed in special education following diagnosis. These results are notable in light of our finding that the sibling cohort is representative of the general population with regard to parent ratings of Externalizing problems (including symptoms of Antisocial, Inattention-Hyperactivity, and Headstrong Behavior). This suggests that these problems do not stem from shared variance associated with a cancer experience. On the other hand, siblings of survivors were more frequently rated as having Internalizing problems (including symptoms of Anxiety-Depression and Social Withdrawal) when compared to the normative expectations, which suggests that siblings of cancer survivors may be at increased risk for affective distress.

As expected, compared to treatment without CRT, treatment including higher doses of CRT conferred increased risk for symptoms of social withdrawal and increased likelihood of special education placement, and even lower doses of CRT conferred increased risk for problems across several domains, including anxiety-depression, inattention-hyperactivity, and social withdrawal. In contrast, higher intensity CRT (i.e., >20 Gy) did not appear to further increase risk for anxiety-depression. This may be due to the challenges associated with the assessment and diagnosis of affective disorders in individuals with significant cognitive impairment.(20-22)

IV-MTX, historically utilized to reduce the need for CRT, conferred increased risk for inattention-hyperactivity, social withdrawal, and special education, even at doses $<4 \cdot 3 \mathrm{~g} / \mathrm{m}^{2}$. This increased risk was present after accounting for age at diagnosis, gender, and treatment with CRT. Further, we found evidence for a dose-response effect, such that survivors treated with higher cumulative doses of IV-MTX were at greater risk for problems with inattentionhyperactivity and were more likely to be placed in special education. These findings are supported by data from a longitudinal study in survivors of childhood ALL treated on a chemotherapy-only protocol, which showed that higher intensity therapy was associated with greater risk for attention problems at the end of therapy and two years post treatment. (23) Significant correlations among survivors treated with CRT and chemotherapy (IT/IVMTX) confounds our ability to analyze the independent contribution of chemotherapy only treatment on outcomes in the current analysis.

Our data also suggest that the onset of neurologic conditions following diagnosis increased risk for adolescent cognitive, behavior, and learning problems. Prior CCSS studies have identified that CRT is a particularly strong risk factor for first and recurrent strokes in survivors. $(24,25)$ Vascular damage imposed by CRT has been associated with stroke in childhood cancer survivors. (26) Survivors of childhood ALL with reported stroke at baseline represented a small percentage of our cohort; nonetheless, the strength of this predictor with regard to adolescent outcomes suggests that these survivors should be monitored closely. 
Importantly, our results suggest that cognitive, behavior and learning problems and participation in special education during adolescence significantly predict early young adult education outcomes. We found that, relative to survivors treated with CRT, survivors treated without CRT reported a higher frequency of college graduation. Regardless of treatment approach, survivors with increased inattention and hyperactivity and those placed in special education were significantly less likely to graduate from college as adults. Parent ratings of inattention and hyperactivity problems were associated with the highest risk for special education placement during adolescence and the highest risk for not graduating from college as adults. These results extend recent findings demonstrating that survivors with attention problems at the end of treatment on a chemotherapy-only protocol perform worse on measures of academic performance two years after completing treatment to now demonstrate a negative impact on long-term educational attainment. (23) In light of these data, interventions in this group should be targeted on remediating or preventing problems during and after therapy.

\section{Limitations}

With the expansion of the CCSS cohort to include survivors diagnosed 1970-1999, the cohort now includes baseline evaluation of the late impact or modern therapy for ALL. However, for our longitudinal analysis we were restricted to examining adult outcomes among survivors treated between 1970 and 1986 who had responded to subsequent followup surveys, which may limit the generalizability of the findings to more recently treated populations. The education level of parents of survivors and siblings was not obtained or available for analyses. Parent education could have an impact on long-term educational outcomes of adolescents. However, as siblings are drawn from families of survivors, it is unlikely to account for differences between survivors and siblings. Our findings are also limited by the lack of self-report data for adolescents at baseline. Future studies may wish to incorporate self-report data for adolescents, as well as performance-based measures of neurocognitive functioning. Given the large sample size we were not able to collect specific information concerning intensity of special education services directly from the adolescent's school. Due to the differences between survivors completing follow-up and those lost to follow-up, our results may be less generalizable to minority populations, adolescents with early indicators of behavior problems, and those treated with higher intensity CNS-directed therapy. We note that this pattern of attrition may lead us to underestimate the strength of the association between predictors and educational outcomes, as the groups that are overrepresented in our lost to follow-up cohort may be at increased risk for lower educational attainment. $(27,28)$

\section{Conclusions}

The current results suggest the need for routine, longitudinal screening in survivors of childhood ALL as they age through adolescence, as problems identified during adolescence are associated with reduced educational outcomes in early young adulthood. Ultimately, early detection of populations at high risk for poor academic outcome will allow for early intervention, in particular for problems with attention and hyperactivity. Increased academic support may also be warranted. A recently completed randomized controlled trial showed that computerized cognitive training is feasible and efficacious for improving existing 
cognitive difficulties in childhood cancer survivors. (29) Future investigations may wish to consider whether prophylactic intervention (i.e., prior to the emergence of cognitive problems) is warranted. Such studies can provide information about whether there is a critical period for intervention efficacy, particularly as over half of children diagnosed with ALL are $\mathbf{5}$ years old and thus may be at increased risk due to vulnerability of developing neural systems. (30)

\section{Supplementary Material}

Refer to Web version on PubMed Central for supplementary material.

\section{Acknowledgments}

Funding: National Cancer Institute (CA55727), Cancer Center Support (CORE) grant (CA21765), and American Lebanese-Syrian Associated Charities (ALSAC).

\section{References}

1. Cancer Facts and Figures. American Cancer Society Incorporated; 2014. Available from: http:// www.cancer.org/research/cancerfactsstatistics/cancerfactsfigures2014/

2. Howlader, N.; Noone, AM.; Krapcho, M.; Garshell, J.; Miller, D.; Altekruse, SF., et al. Institue NC. SEER Cancer Statistics Review, 1975-2012. Bethesda, MD: National Cancer Institue; 2015.

3. Pui CH, Evans WE. A 50-year journey to cure childhood acute lymphoblastic leukemia. Seminars in hematology. 2013; 50(3):185-96. [PubMed: 23953334]

4. Cheung YT, Krull KR. Neurocognitive outcomes in long-term survivors of childhood acute lymphoblastic leukemia treated on contemporary treatment protocols: A systematic review. Neuroscience and biobehavioral reviews. 2015; 53:108-20. [PubMed: 25857254]

5. Iyer NS, Balsamo LM, Bracken MB, Kadan-Lottick NS. Chemotherapy-only treatment effects on long-term neurocognitive functioning in childhood ALL survivors: a review and meta-analysis. Blood. 2015; 126(3):346-53. [PubMed: 26048910]

6. Kunin-Batson A, Kadan-Lottick N, Neglia JP. The contribution of neurocognitive functioning to quality of life after childhood acute lymphoblastic leukemia. Psycho-oncology. 2014; 23(6):692-9. [PubMed: 24497266]

7. Buizer AI, de Sonneville LM, van den Heuvel-Eibrink MM, Veerman AJ. Chemotherapy and attentional dysfunction in survivors of childhood acute lymphoblastic leukemia: effect of treatment intensity. Pediatr Blood Cancer. 2005; 45(3):281-90. [PubMed: 15806539]

8. Conklin HM, Krull KR, Reddick WE, Pei D, Cheng C, Pui CH. Cognitive outcomes following contemporary treatment without cranial irradiation for childhood acute lymphoblastic leukemia. $\mathrm{J}$ Natl Cancer Inst. 2012; 104(18):1386-95. [PubMed: 22927505]

9. Buizer AI, De Sonneville LM, van den Heuvel-Eibrink MM, Njiokiktjien C, Veerman AJ. Visuomotor control in survivors of childhood acute lymphoblastic leukemia treated with chemotherapy only. Journal of the International Neuropsychological Society: JINS. 2005; 11(5): 554-65. [PubMed: 16212682]

10. Kingma A, van Dommelen RI, Mooyaart EL, Wilmink JT, Deelman BG, Kamps WA. Slight cognitive impairment and magnetic resonance imaging abnormalities but normal school levels in children treated for acute lymphoblastic leukemia with chemotherapy only. The Journal of pediatrics. 2001; 139(3):413-20. [PubMed: 11562622]

11. von der Weid N, Mosimann I, Hirt A, Wacker P, Beck MN, Imbach P, et al. Intellectual outcome in children and adolescents with acute lymphoblastic leukaemia treated with chemotherapy alone: age- and sex-related differences. Eur J Cancer. 2003; 39:359-65. [PubMed: 12565989]

12. Jain N, Brouwers P, Okcu MF, Cirino PT, Krull KR. Sex-specific attention problems in long-term survivors of pediatric acute lymphoblastic leukemia. Cancer. 2009; 115(18):4238-45. [PubMed: 19536898] 
13. Blakemore SJ. Development of the social brain during adolescence. Quarterly journal of experimental psychology. 2008; 61(1):40-9.

14. Sisk CL, Foster DL. The neural basis of puberty and adolescence. Nature neuroscience. 2004; 7(10):1040-7. [PubMed: 15452575]

15. Gerhardt CA, Lehmann V, Long KA, Alderfer MA. Supporting Siblings as a Standard of Care in Pediatric Oncology. Pediatric blood \& cancer. 2015; 62(Suppl 5):S750-804. [PubMed: 26700924]

16. Robison LL, Armstrong GT, Boice JD, Chow EJ, Davies SM, Donaldson SS, et al. The Childhood Cancer Survivor Study: a National Cancer Institute-supported resource for outcome and intervention research. Journal of clinical oncology: official journal of the American Society of Clinical Oncology. 2009; 27(14):2308-18. [PubMed: 19364948]

17. Leisenring WM, Mertens AC, Armstrong GT, Stovall MA, Neglia JP, Lanctot JQ, et al. Pediatric cancer survivorship research: experience of the Childhood Cancer Survivor Study. Journal of clinical oncology: official journal of the American Society of Clinical Oncology. 2009; 27(14): 2319-27. [PubMed: 19364957]

18. Peterson JL, Zill N. Marital Disruption, Parent-Child Relationships, and Behavior Problems in Children. Journal of Marriage and Family. 1986; 48(2):295-307.

19. Krull KR, Brinkman TM, Li C, Armstrong GT, Ness KK, Srivastava DK, et al. Neurocognitive outcomes decades after treatment for childhood acute lymphoblastic leukemia: a report from the St Jude lifetime cohort study. J Clin Oncol. 2013; 31(35):4407-15. [PubMed: 24190124]

20. Esbensen AJ, Seltzer MM, Greenberg JS, Benson BA. Psychometric evaluation of a self-report measure of depression for individuals with mental retardation. American journal of mental retardation: AJMR. 2005; 110(6):469-81. [PubMed: 16212449]

21. Seltzer MM, Floyd F, Greenberg J, Lounds J, Lindstromm M, Hong J. Life course impacts of mild intellectual deficits. American journal of mental retardation: AJMR. 2005; 110(6):451-68. [PubMed: 16212448]

22. Marston GM, Perry DW, Roy A. Manifestations of depression in people with intellectual disability. Journal of intellectual disability research: JIDR. 1997; 41(Pt 6):476-80. [PubMed: 9430051]

23. Jacola LM, Krull KR, Pui C-H, Pei D, Cheng C, Reddick WE, et al. Longitudinal assessment of neurocognitive outcomes in survivors of childhood acute lymphoblastic leukemia treated on a contemporary chemotherapy protocol. Journal of Clinical Oncology. 2016; 34(11):1239-47. [PubMed: 26858334]

24. Bowers DC, Liu Y, Leisenring W, McNeil E, Stovall M, Gurney JG, et al. Late-occurring stroke among long-term survivors of childhood leukemia and brain tumors: a report from the Childhood Cancer Survivor Study. J Clin Oncol. 2006; 24(33):5277-82. [PubMed: 17088567]

25. Fullerton HJ, Stratton K, Mueller S, Leisenring WW, Armstrong GT, Weathers RE, et al. Recurrent stroke in childhood cancern survivors. Neurology. 2015; 85:1056-64. [PubMed: 26311747]

26. Mueller S, Fullerton HJ, Stratton K, Leisenring W, Weathers RE, Stovall M, et al. Radiation, atherosclerotic risk factors, and stroke risk in survivors of pediatric cancer: a report from the Childhood Cancer Survivor Study. International journal of radiation oncology, biology, physics. 2013; 86(4):649-55.

27. Mitby PA, Robison LL, Whitton JA, Zevon MA, Gibbs IC, Tersak JM, et al. Utilization of special education services and educational attainment among long-term survivors of childhood cancer: a report from the Childhood Cancer Survivor Study. Cancer. 2003; 97(4):1115-26. [PubMed: 12569614]

28. Rising Tide: Do College Grad Rate Gains Benefit All Students? Report published by the Education Trust. Dec. 2015 https://edtrust.org/wp-content/uploads/2014/09/TheRisingTide-Do-College-GradRate-Gains-Benefit-All-Students-3.7-16.pdf

29. Conklin H, Ogg RJ, Ashford JA, Scoggins MA, Zou P, Clark KN, Martin-Elbahesh K, Hardy KK, Merchant TE, Jeha S, Huang L, Zhang H. Computerized Cognitive Training for Amelioration of Cognitive Late Effects among Childhood Cancer Survivors: A Randomized Controlled Trial. Journal of clinical oncology: official journal of the American Society of Clinical Oncology. 2015

30. Lebel C, Walker L, Leemans A, Phillips L, Beaulieu C. Microstructural maturation of the human brain from childhood to adulthood. NeuroImage. 2008; 40(3):1044-55. [PubMed: 18295509] 


\section{Panel: Research in context}

\section{Evidence before this study}

The replacement of cranial radiation therapy (CRT) with intensified systemic and intrathecal chemotherapy for CNS prophylaxis in the treatment of childhood acute lymphoblastic leukemia (ALL) has resulted in improved global cognitive outcomes; still, a review of neurocognitive studies in survivors treated without CRT have suggested increased risk for deficits in attention and higher-order cognitive skills. The predictors and impact of these deficits on long-term functional outcomes is not well established, and few longitudinal studies exist to examine the trajectory of neurocognitive functioning over time. Our literature search was conducted PubMed with the following combination of keywords: "acute lymphoblastic leukemia", "childhood", "pediatric", "behavioral", "psychological”, "neurocognitive", "attention", "learning", "education outcomes", "executive function", and "processing speed". We restricted our search to articles available in English and published between 1 January 2001 and 30 April 2016.

\section{Added value of this study}

The current study used data from the multi-institutional Childhood Cancer Survivor Study (CCSS) to identify the prevalence and predictors of parent-reported cognitive, behavior, and learning problems in 1560 adolescent survivors of ALL diagnosed between 1970 and 1999, thus allowing us to evaluate the impact of modern ALL therapy. Compared to survivors treated with CRT, the frequency of cognitive, behavior, and learning problems in adolescent and early young adult survivors treated on chemotherapy-only protocols is significantly lower, reflecting the relative preservation of neurobehavioral outcomes in survivors treated with contemporary therapy. Nonetheless, we found that a significant proportion of survivors treated without radiation still experience problems with anxiety-depression, inattention-hyperactivity, and social withdrawal compared to sibling controls. These problems were associated with concurrent learning difficulties and predicted reduced adult educational attainment. Notably, we found that inattention and hyperactivity problems were associated with the highest risk for special education placement during adolescence and the highest risk for not graduating from college as adults, and that participation in special education during adolescence did not improve adult educational attainment.

\section{Implications of all the available evidence}

The current results suggest the need for routine, longitudinal screening in survivors of childhood ALL as they age through adolescence, as problems identified during adolescence are associated with reduced educational outcomes in early young adulthood. Identification of cognitive and behavioral problems during adolescent should be treated with targeted therapeutic interventions and not just special education placement in the school system. 


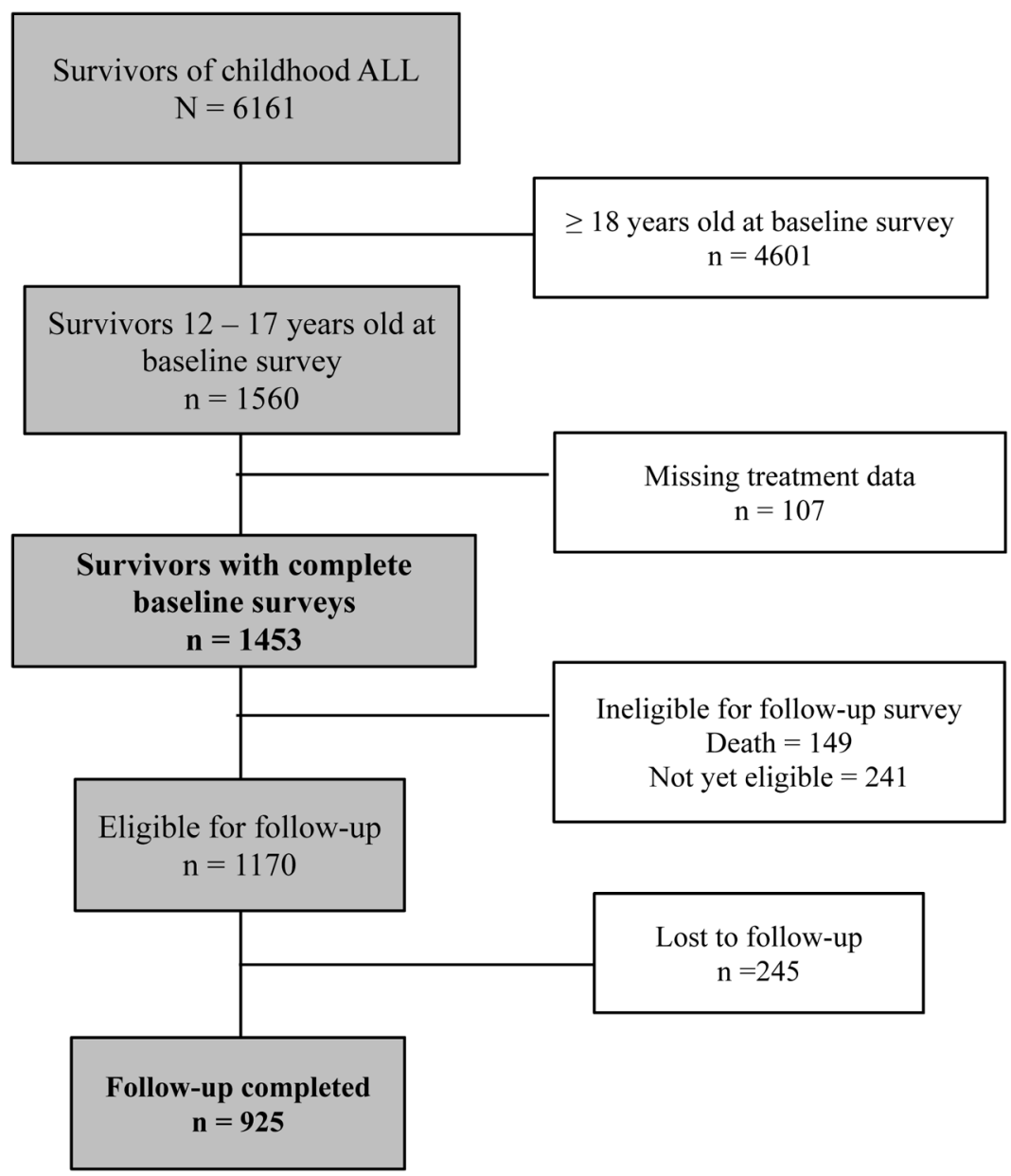

Figure 1.

Consort diagram for survivor cohort 

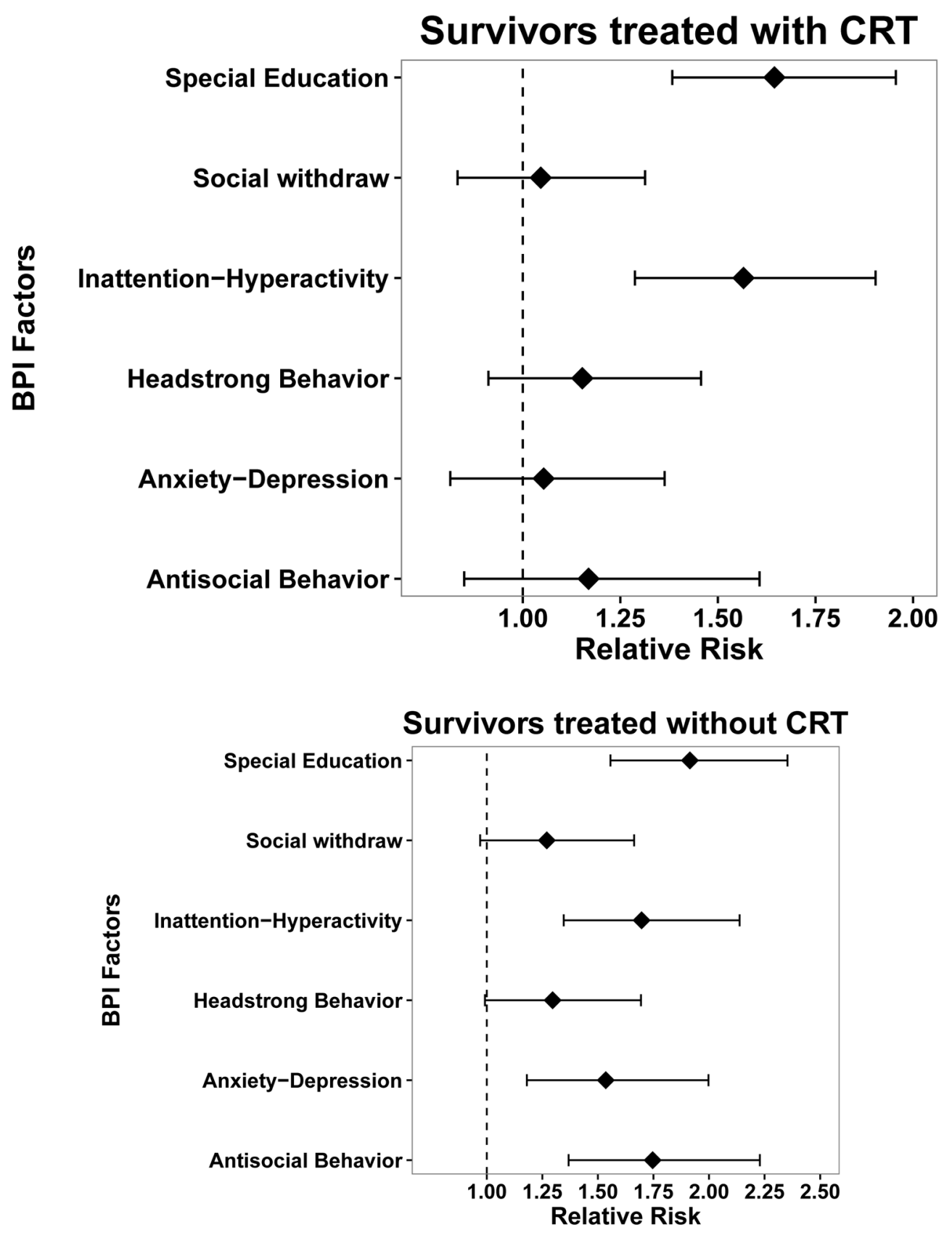

Figure 2.

Poisson regression examining the impact of cognitive and behavior problems on reduced attainment of college education in survivors with and without CRT 


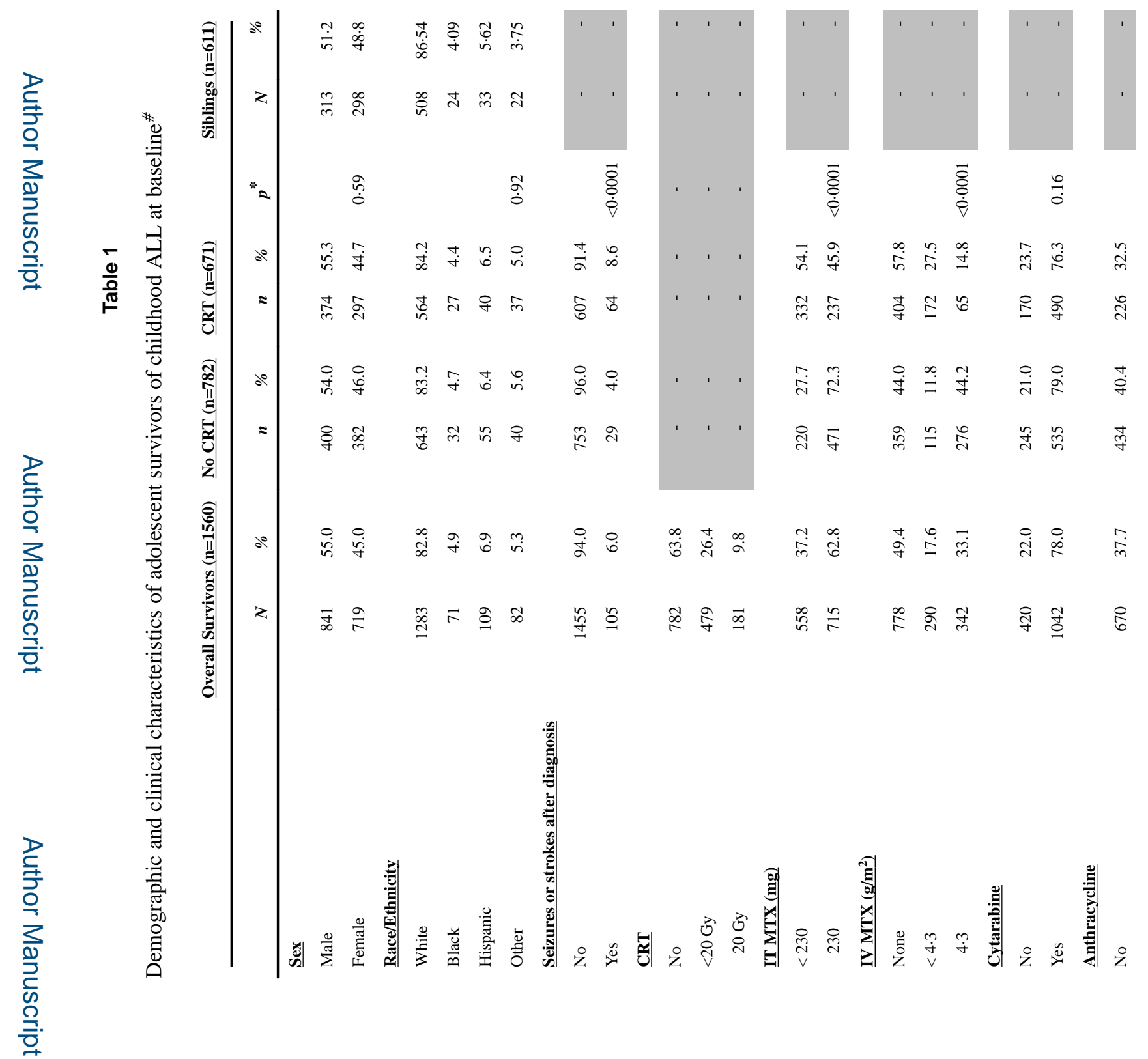
Lancet Psychiatry. Author manuscript; available in PMC 2017 October 01. 


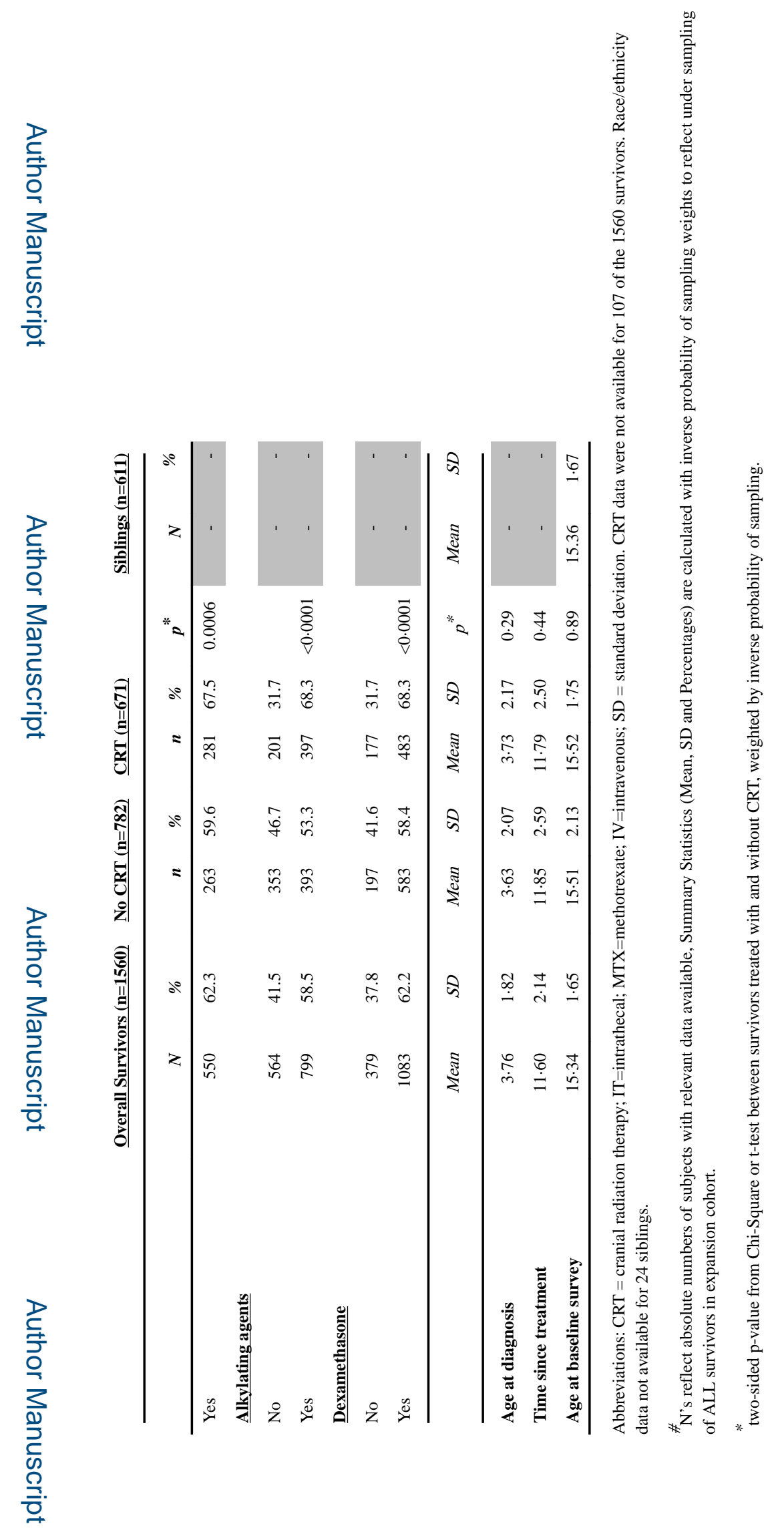




\section{롤 \\ 일}

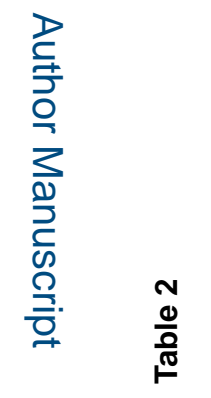

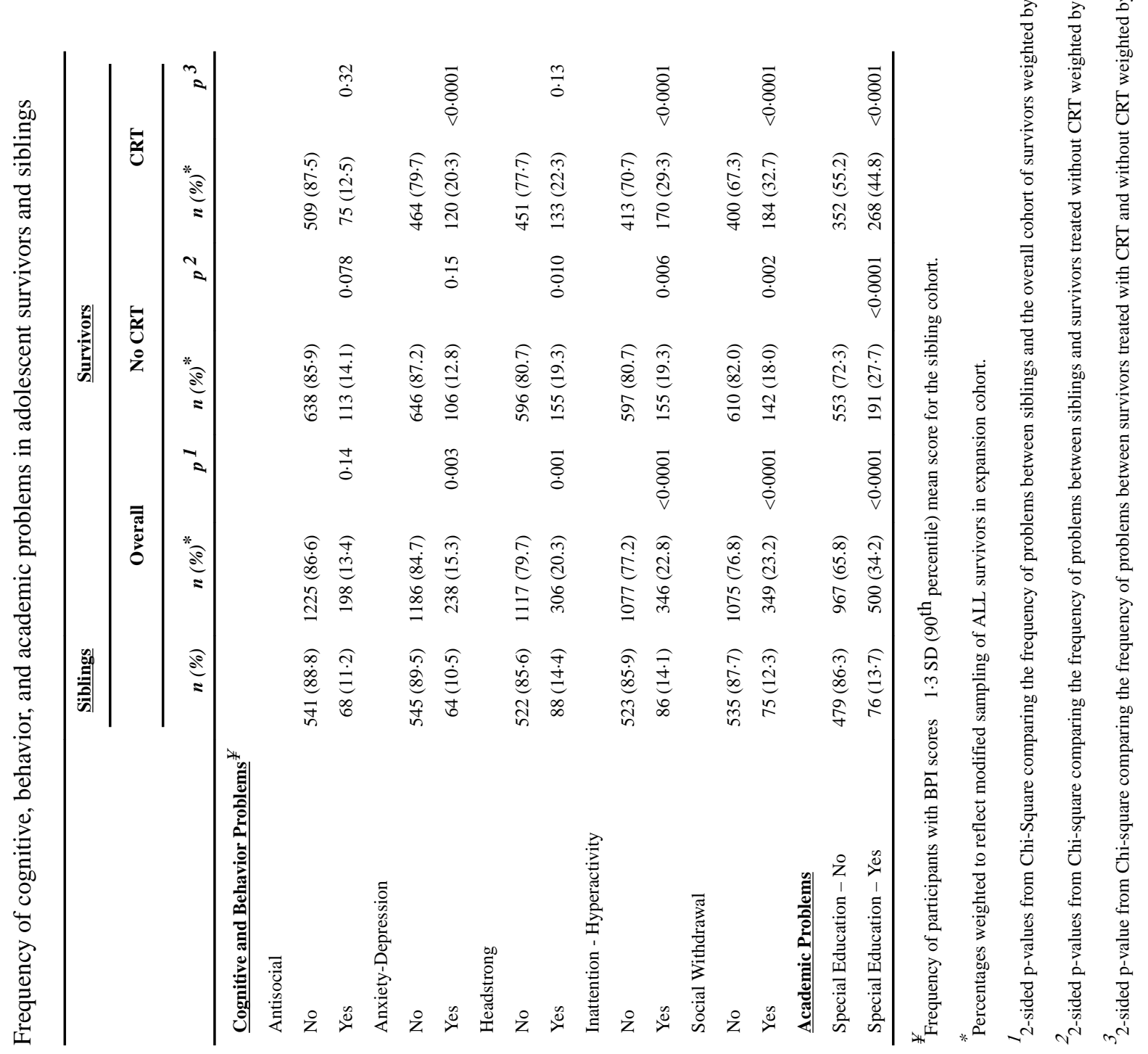

Lancet Psychiatry. Author manuscript; available in PMC 2017 October 01. 


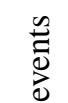
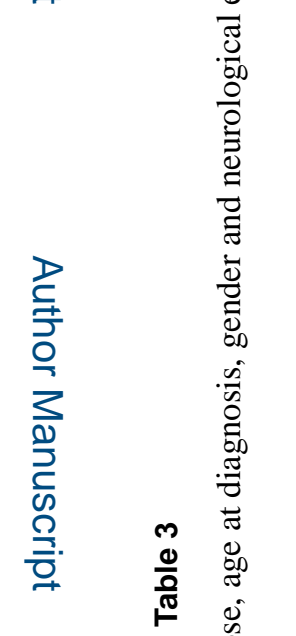

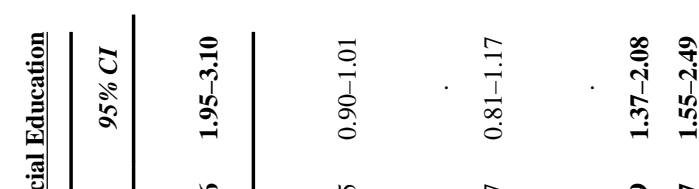

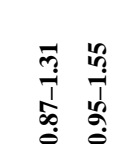

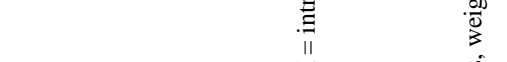

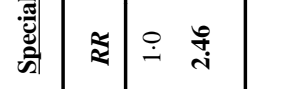

ถู

$-\hat{a}$

$-\stackrel{a}{9}$

$\stackrel{9}{\text { ติ }}$

$\stackrel{9}{9}$

$\stackrel{9}{9}$

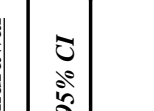



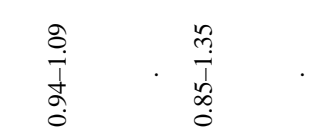

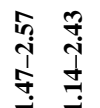

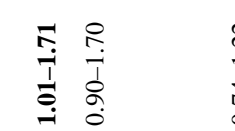

莒

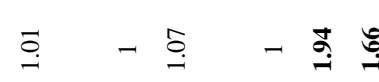

$\stackrel{-}{\dddot{m}} \stackrel{ \pm}{I}$

$\stackrel{\circ}{\circ}$

.

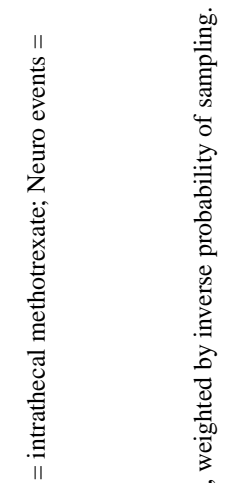

|

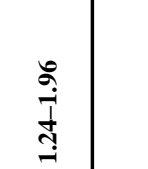

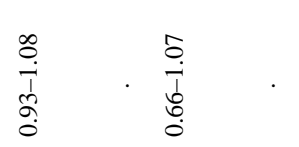

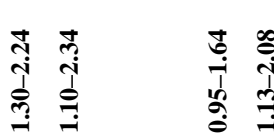

$\stackrel{\circ}{\infty} \stackrel{\infty}{-}$

$\mid$\begin{tabular}{l}
$\sum_{i}$ \\
\hline
\end{tabular}

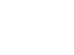

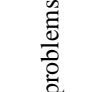

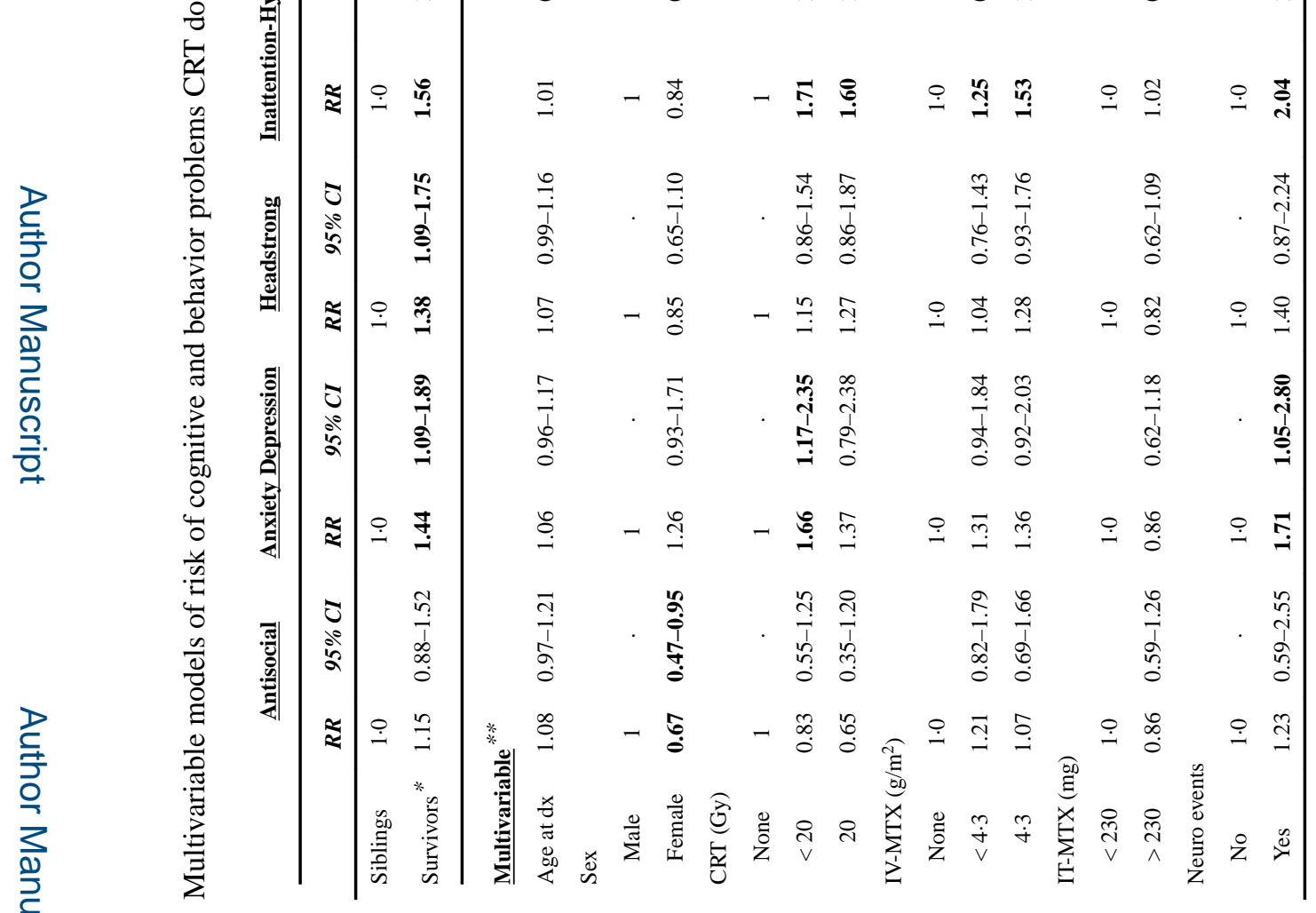

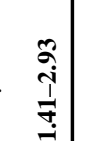

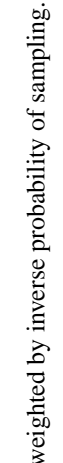

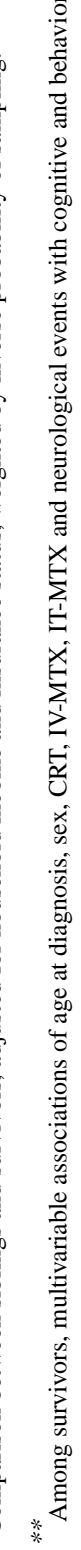

Lancet Psychiatry. Author manuscript; available in PMC 2017 October 01. 